\title{
Broadening the phenotype of TARDBP mutations: the TARDBP Ala382Thr mutation and Parkinson's disease in Sardinia
}

\author{
Marialuisa Quadri • Giovanni Cossu • Valeria Saddi • \\ Erik J. Simons • Daniela Murgia • Maurizio Melis • \\ Anna Ticca $\cdot$ Ben A. Oostra $\cdot$ Vincenzo Bonifati
}

Received: 19 April 2011 /Accepted: 24 May 2011 /Published online: 11 June 2011

(C) The Author(s) 2011. This article is published with open access at Springerlink.com

\begin{abstract}
Mutations in the TARDBP gene are a cause of autosomal dominant amyotrophic lateral sclerosis (ALS) and of frontotemporal lobar degeneration (FTLD), but they have not been found so far in patients with Parkinson's disease (PD). A founder TARDBP mutation (p.Ala382Thr) was recently identified as the cause of $\sim 30 \%$ of ALS cases in Sardinia, a Mediterranean genetic isolate. We studied 327 consecutive Sardinian patients with clinically diagnosed PD (88 familial, 239 sporadic) and 578 Sardinian controls. One family with FTLD and parkinsonism was also included. The p.Ala382Thr heterozygous mutation was detected in eight unrelated PD patients $(2.5 \%)$. The three patients from the FTLD/parkinsonism family also carried this mutation. Within the control group, there were three heterozygous mutation carriers. During follow-up, one of these individuals developed motoneuron disease and another, a rapidly progressive dementia; the third remains healthy at the age of 79 but two close relatives developed motoneuron disease and dementia. The eight PD patients carrying the $\mathrm{p}$. Ala382Thr mutation had all sporadic disease presentation.
\end{abstract}

M. Quadri · E. J. Simons • B. A. Oostra • V. Bonifati $(\bowtie)$

Department of Clinical Genetics, Erasmus MC, P.O. Box 2040, 3000 CA Rotterdam, The Netherlands e-mail: v.bonifati@erasmusmc.nl

G. Cossu $(\bowtie) \cdot$ D. Murgia $\cdot$ M. Melis

Neurology Service and Stroke Unit,

General Hospital S. Michele AOB "G. Brotzu”,

Piazzale Ricchi 1,

09134 Cagliari, Italy

e-mail: giovannicossu@aob.it

V. Saddi $\cdot$ A. Ticca

Neurology Division, S. Francesco Hospital,

ASL No. 3 Nuoro, Italy
Their average onset age was 70.0 years (SD 9.4, range 5179), which is later but not significantly different from that of the patients who did not carry this mutation. In conclusion, we expand the clinical spectrum associated with TARDBP mutations to FTLD with parkinsonism without motoneuron disease and to clinically definite PD. The TDP-43 protein might be directly involved in a broader neurodegenerative spectrum, including not only motoneuron disease and FTLD but also PD.

Keywords Parkinson's disease - Frontotemporal lobar degeneration · Amyotrophic lateral sclerosis · Sardinia . TARDBP. Mutation

\section{Introduction}

The identification of the "transactive response (TAR) DNAbinding protein-43" (TDP-43) as the main component of the pathological lesions in patients with frontotemporal lobar degeneration (FTLD) with ubiquitin-positive inclusions, in those with amyotrophic lateral sclerosis (ALS), and in FTLD with motoneuron disease (FTLD-MND) provided strong support to the contention that these seemingly different diseases represent different parts of one neurodegenerative spectrum [1]. Soon thereafter, missense mutations in the gene encoding the TDP-43 protein $(T A R D B P)$ were identified in patients with autosomal dominant ALS $[2,3]$ and, albeit more rarely, in patients with FTLD with or without ALS [4-6], demonstrating a primary, causal role for the TDP-43 protein abnormalities in the pathogenesis of these diseases, now also termed TDP43 proteinopathies [7]. The function of the TDP-43 protein remains poorly understood, but it has been initially 
characterized as a transcription factor which binds to TAR DNA sequence motif [8] and, later, as a modulator of the alternative mRNA splicing [9].

Several TARDBP mutations have been identified in patients with familial and also sporadic ALS, these latter pointing to an incomplete, age-related penetrance [10-17]. The c.G1144A substitution (predicted protein effect: $p$. Ala382Thr) is the most frequent TARDBP mutation known so far, which has been found in ALS patients of Italian and French origins $[3,15,16]$. Very recently, the same missense mutation was identified as the disease cause in a large percentage $(\sim 30 \%)$ of familial and sporadic ALS patients from Sardinia, a genetically isolated Mediterranean island $[18,19]$. Haplotype analyses in the Sardinian, Italian, and French cases strongly suggested that this mutation originated from a common founder $[15,18,19]$.

The occurrence of parkinsonism and motoneuron disease in the same patient has sometimes been reported [20]. Of note, three Sardinian patients with ALS who carried the TARDBP p.Ala382Thr mutation developed parkinsonism after the onset of the motoneuron disease [18]. However, to our knowledge, TARDBP mutations have not been described in patients with Parkinson's disease (PD).

As part of an ongoing research program on the genetics of PD in Sardinia, we therefore investigated the occurrence of the TARDBP p.Ala382Thr mutation among patients with this disease from this genetically isolated population. We detected 8 out of the 327 PD cases $(2.5 \%)$ who are carriers of this mutation. Our data provide therefore a first direct link between the TARDBP mutation and PD, supporting an involvement of the TDP-43 protein in a broader neurodegenerative spectrum, including not only motoneuron disease and FTLD, but also PD.

\section{Subjects and methods}

All cases and controls included in the study (905 subjects) were born and living in Sardinia, and all their four grandparents were also born in Sardinia. The project was approved by the relevant ethical authorities and written informed consent was obtained from all participating subjects.

The PD group included 327 consecutive patients (125 females, 202 males) with clinically diagnosed PD, recruited at two movement disorder units in southern central areas of Sardinia: the General Hospital "S. Michele" in Cagliari (301 patients) and the S. Francesco Hospital in Nuoro (26 patients). The average onset age was 62.6 years (SD 10.5, range 22-84). Additional clinical features are shown in Table 1. All patients fulfilled the United Kingdom Brain Bank Criteria for the clinical diagnosis of PD [21]. No pathological studies were available. A detailed medical
Table 1 Clinical features in the PD series

\begin{tabular}{lll}
\hline p.Ala382Thr & Het carriers & Non-carriers \\
\hline Total no. of patients & 8 & 319 \\
Males & 7 & 195 \\
Females & 1 & 124 \\
Familial PD & 0 & 88 \\
Sporadic PD & 8 & 231 \\
PD onset age, years [average (SD)] & $70.0(9.4)$ & $62.9(10.5)$ \\
\hline
\end{tabular}

history of the nuclear family was recorded from all the PD cases. Patients were classified as sporadic if they reported no other PD cases among first- and second-degree relatives. Accordingly, there were 88 patients with familial and 239 with sporadic PD presentation. The clinical evaluation of the parkinsonism was performed using the Hoehn-Yahr Scale and the Unified Parkinson's Disease Rating Scale (UPDRS) [22]. The Mini-Mental State Examination (MMSE) was used as a screening test for the cognitive status.

One family with three siblings affected by different combinations of atypical parkinsonism, FTLD, and L-doparesponsive parkinsonism was also ascertained and studied (Fig. 1). A first control group consisted of 203 subjects, age-matched to the patients ( 86 females, 117 males), mainly spouses of PD cases or subjects examined at the same centers for diseases unrelated to PD. The average age at sampling was 71.3 years (SD 10.8, range 38-93). Among these, 174 were collected in Cagliari and 29 in Nuoro. Controls were eligible for inclusion only if they reported a negative family history of PD among first- and seconddegree relatives. Moreover, at the time of the examination, none of the controls showed any signs or symptoms of parkinsonism, dementia, motoneuron disease or other neurodegenerative diseases.

A second, younger group of controls was available, consisting of 375 healthy subjects ( 92 females, 283 males) ascertained within a research program on the genetics of multiple sclerosis in Sardinia [23]. The average age at sampling of this second control group was 42.8 years (SD 10.6, range 21-66).

Genomic DNA was isolated from peripheral blood using standard protocols. In the whole case-control sample, the presence of the TARDBP c.G1144A mutation (p.Ala382Thr) was assessed by direct DNA sequencing. In patients who carried this mutation and in additional selected patients with familial PD, the whole TARDBP coding region and exonintron boundaries was sequenced. In all cases with the TARDBP p.Ala382Thr mutation, we also searched for the LRRK2 p.Gly2019Ser mutation (previously reported to occur, albeit rarely, among Sardinian PD patients) [24] by direct sequencing, as previously described [24]. 
Fig. 1 Genetic findings and neuroimaging in a family with FTLD with parkinsonism and $\mathrm{PD}$. a Pedigree of the family. b Electropherogram showing the heterozygous TARDBP p.Ala382Thr mutation. c DaTSCAN-SPECT in the SAR-403 patient showing severe defects at the level of the caudate nucleus and putamen, bilaterally

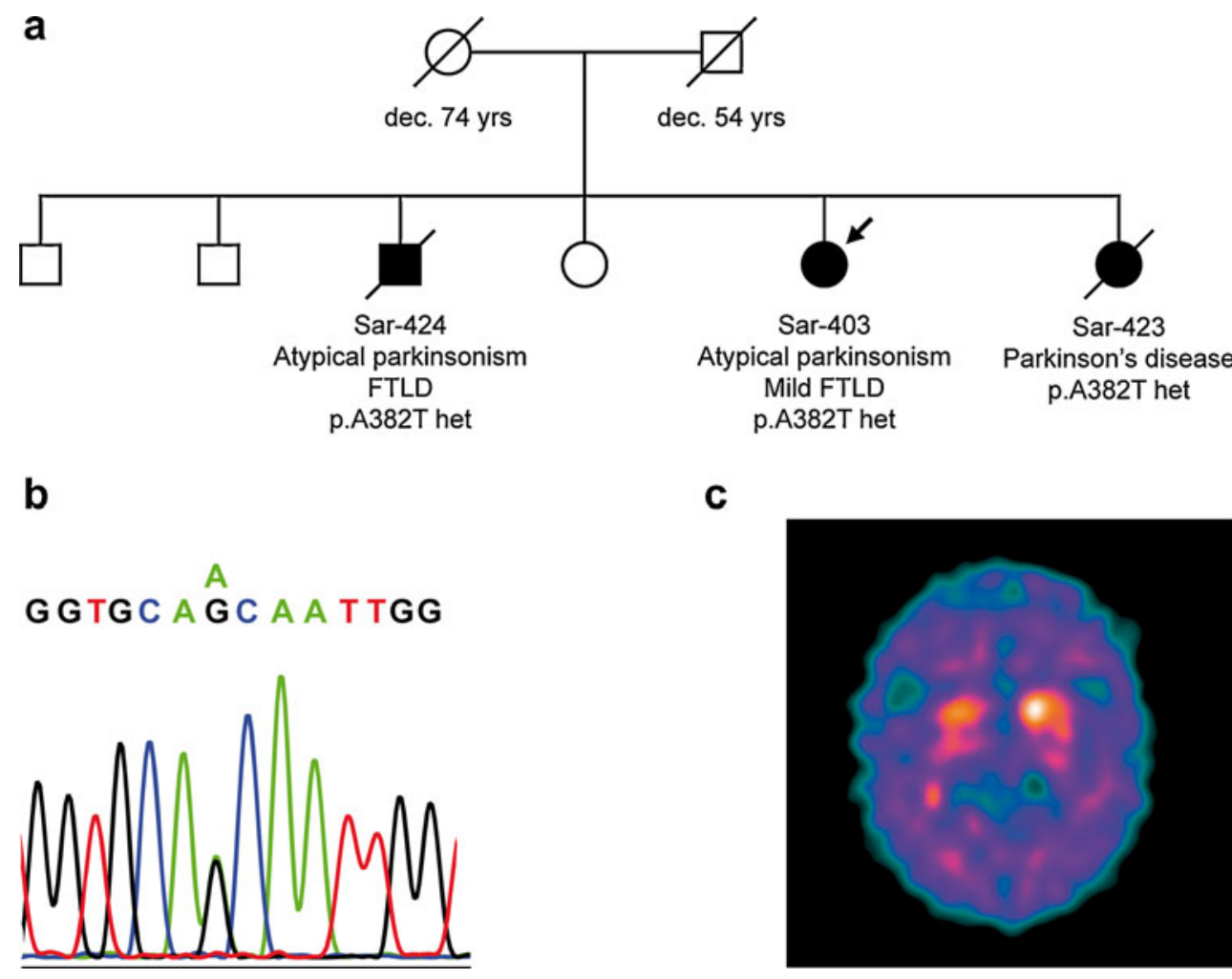

Fragments were amplified by PCR (protocols and primers available on request from the authors). Direct sequencing was performed using Big Dye terminator chemistry ver.3.1 (Applied Biosystem). Fragments were loaded on an Applied Biosystem 3130XL automated sequencer and analyzed with DNA Sequencing analysis (ver.5.3) and SeqScape (ver.2.6) software (Applied Biosystem). The TARDBP mutation is numbered from the "A" of the ATG-translation initiation codon, according to GenBank reference sequences, accession number NM_007375.3.

\section{Results}

We identified 8 PD cases who carried the TARDBP c. G1144A (p.Ala382Thr) variant in heterozygous state (8/ $327,2.5 \%)$ and no homozygous carriers. The detailed clinical features in the patients who carried the p.Ala382Thr mutation are shown in Table 2. Sequencing the whole TARDBP coding region and exon-intron boundaries in patients with the p.Ala382Thr mutation, as well as in five additional patients with familial PD revealed no additional

Table 2 Clinical features in the PD patients carrying the TARDBP p.Ala382Thr mutation

\begin{tabular}{|c|c|c|c|c|c|c|c|c|c|c|}
\hline Patient & $\begin{array}{l}\text { Familial/ } \\
\text { sporadic }\end{array}$ & $\begin{array}{l}\text { Age } \\
\text { (years) }\end{array}$ & Gender & $\begin{array}{l}\text { PD onset } \\
\text { (years) }\end{array}$ & $\begin{array}{l}\text { Disease } \\
\text { duration } \\
\text { (years) }\end{array}$ & $\begin{array}{l}\text { Initial symptoms } \\
\text { (asymmetry) }\end{array}$ & L-dopa resp. & DaTSCAN & $\begin{array}{l}\text { Supportive non- } \\
\text { motor symptoms }\end{array}$ & $\begin{array}{l}\text { MMSE } \\
\text { score }\end{array}$ \\
\hline Sar-006 & Sporadic & 86 & M & 79 & 7 & RT, B, R (right) & Sustained & n.p. & Hyposmia & $24 / 30$ \\
\hline Sar-053 & Sporadic & 65 & M & 51 & 14 & RT (right) & Sustained & n.p. & $\mathrm{RBD}$ & $30 / 30$ \\
\hline Sar-104 & Sporadic & 71 & M & 65 & 6 & RT, B, R (right) & Sustained & Typical for PD & RBD, hyposmia & $27 / 30$ \\
\hline Sar-146 & Sporadic & 84 & M & 78 & 6 & RT, B, R (right) & Sustained & n.p. & RBD, hyposmia & $23 / 30$ \\
\hline Sar-171 & Sporadic & 80 & M & 75 & 5 & $\begin{array}{l}\text { RT, B, R (right) mild } \\
\text { cognitive decline }\end{array}$ & + & n.p. & - & $18 / 30$ \\
\hline Sar-220 & Sporadic & 91 & M & 71 & 20 & B, R (right) & + & n.p. & - & $22 / 30$ \\
\hline Sar-239 & Sporadic & 71 & $\mathrm{~F}$ & 65 & 6 & RT, B, R (right) & Sustained & Typical for PD & RBD & $29 / 30$ \\
\hline Sar-579 & Sporadic & 81 & M & 76 & 5 & $\begin{array}{l}\text { RT, B, R (left) mild } \\
\text { cognitive decline }\end{array}$ & + & n.p. & - & $20 / 30$ \\
\hline
\end{tabular}

$M$ male, $F$ female, $R T$ rest tremor, $B$ bradykinesia, $R$ rigidity, MMSE Mini-Mental State Examination, n.p. not performed, $R B D$ REM-sleep behavior disorder, + present, - absent 
novel variants. No carriers of the LRRK2 Gly2019Ser mutation were detected.

Within the first group of controls, we identified three subjects who also carried the p.Ala382Thr mutation in heterozygous state and none who carried it in homozygous state. Within the second group of controls, no mutation carriers were detected. Our follow-up revealed that one of the three heterozygous mutation carriers had developed motoneuron disease at age 61, 2 years after sampling; another developed a rapidly progressive dementia starting approximately at age 81, 1 year after sampling, and he has became bedridden, anarthric, and totally dependent from caregivers' assistance within 4 years of disease evolution. The third subject remains free from signs or symptoms of motoneuron disease, dementia, or parkinsonism at the age of 79, but two of his close relatives have recently developed motoneuron disease and dementia. If one excludes these three control subjects who developed motoneuron disease, or dementia, or who had close relatives with these diseases, none of the 575 Sardinian controls in this study $(1,150$ chromosomes) carry the p.Ala382Thr mutation. Furthermore, in previous studies, this mutation was not detected in more than 500 Sardinian control chromosomes $[18,19]$ and in more than 1,900 Italian control chromosomes $[15,16]$.

None of the eight PD patients who carried the $p$. Ala382Thr mutation reported any first- or second-degree relatives affected by PD, dementia, or motoneuron disease. They are therefore classified as sporadic PD according to our criteria. However, information on parental age at death was available for only six of the eight mutation carrier probands (Table 3). Considering the late onset age of PD in these cases and the parental age at death, censoring effects might have masked the familial nature of the disease in at least some of these cases. Furthermore, two patients (Sar006 and Sar-104) reported each one more distant relative (first cousin) with parkinsonism. Our examination of these distant relatives revealed in both cases a late onset, atypical parkinsonism with poor response to levodopa, and genetic

Table 3 Age at death among the parents of PD patients carrying the $p$. Ala382Thr mutation

\begin{tabular}{llll}
\hline PD patient & Mother & Father & PD onset in offspring \\
\hline Sar-006 & 85 & 80 & 79 \\
Sar-053 & n.a. & n.a. & 51 \\
Sar-104 & $72^{\text {a }}$ & 84 & 65 \\
Sar-146 & 80 & 90 & 78 \\
Sar-171 & 83 & 30 & 75 \\
Sar-220 & n.a. & n.a. & 71 \\
Sar-239 & 64 & 64 & 65 \\
Sar-579 & 87 & 60 & 76 \\
\hline
\end{tabular}

${ }^{\mathrm{a}}$ Referred with tremor testing revealed absence of the p.Ala382Thr mutation. We consider these two distant relatives as phenocopies.

The eight patients who carried the p.Ala382Thr mutation all fulfilled the UK PD Society Brain Bank criteria for a clinical diagnosis of definite PD. Six of these display a classical PD presentation (Table 2), while two (Sar-171 and Sar-579) present with a postural instability-gait disturbance predominant type of PD and also have mild cognitive impairment and psychotic phenomena.

The average PD symptoms onset in the eight carriers of the p.Ala382Thr mutation was $70.0 \pm 9.4$ years (range $51-$ 79), which is later though not significantly different from the average onset age among the patients who did not carry this mutation $(62.9 \pm 10.5$ years, range $22-83, p=0.08)$. Brain SPECT images (DaTSCAN) were available from two patients with the p.Ala382Thr mutation and are shown in Fig. 2.

Within the FTLD-parkinsonism family (Fig. 1), three siblings were affected by different combinations of atypical parkinsonism, FTLD, and L-dopa-responsive parkinsonism, and they all carried the p.Ala382Thr heterozygous mutation. The patients' mother died at the age of 74 and the father at the age of 54 (due to hepatic disease), without evidence of neurodegenerative diseases. The proband (Sar403 ) is a 77-year-old, congenital deaf-mute woman, who developed resting tremor and bradykinesia in her right limbs and at the age of 76. At the moment of our examination, after 1 year of disease course, her H-Y stage was 1.5 and the UPDRS motor score 13. Only mild benefit was observed after 12 months of L-dopa treatment (daily dose of $500 \mathrm{mg}$, four times per day). Though her MMSE was 30/30, she developed hallucinations and mild cognitive disturbances of frontal lobe type. Brain CT scan was unremarkable, but DaTSCAN showed severe bilateral dopaminergic deficiency at the level of both putamen and caudate nuclei (Fig. 1c), suggestive of an atypical parkinsonian disorder. Of note, evidence of motoneuron disease was not detected after both clinical examination and EMG investigations, after 1 year of disease course.

The proband's brother (Sar-424) developed a rapidly progressive, symmetrical akinetic-rigid parkinsonism with onset at the age of 68 years, with only a mild response to Ldopa treatment (daily dose of $600 \mathrm{mg}$, four times per day). At the moment of our examination, after 2 years of disease course, his H-Y stage was 4, the UPDRS motor score was 54, and the MMSE was 28/30, but he also showed severe dementia of frontotemporal type. Frontotemporal atrophy was also seen in his brain MRI. He died 2 years later at the age of 72 .

Interestingly, the proband's sister (Sar-423) developed symmetrical akinetic-rigid parkinsonism without resting tremor at the age of 74 years. At the moment of our examination, after 1 year of disease course, her H-Y stage 
Fig. 2 DaTSCAN-SPECT images in PD patients with the TARDBP p.Ala382Thr mutation. In two patients carrying the TARDBP p.Ala382Thr mutation (a Sar-239, b Sar-104), the DaTSCAN-SPECT imaging shows asymmetric defects, predominantly at the level of the putamen, as typically observed in idiopathic PD
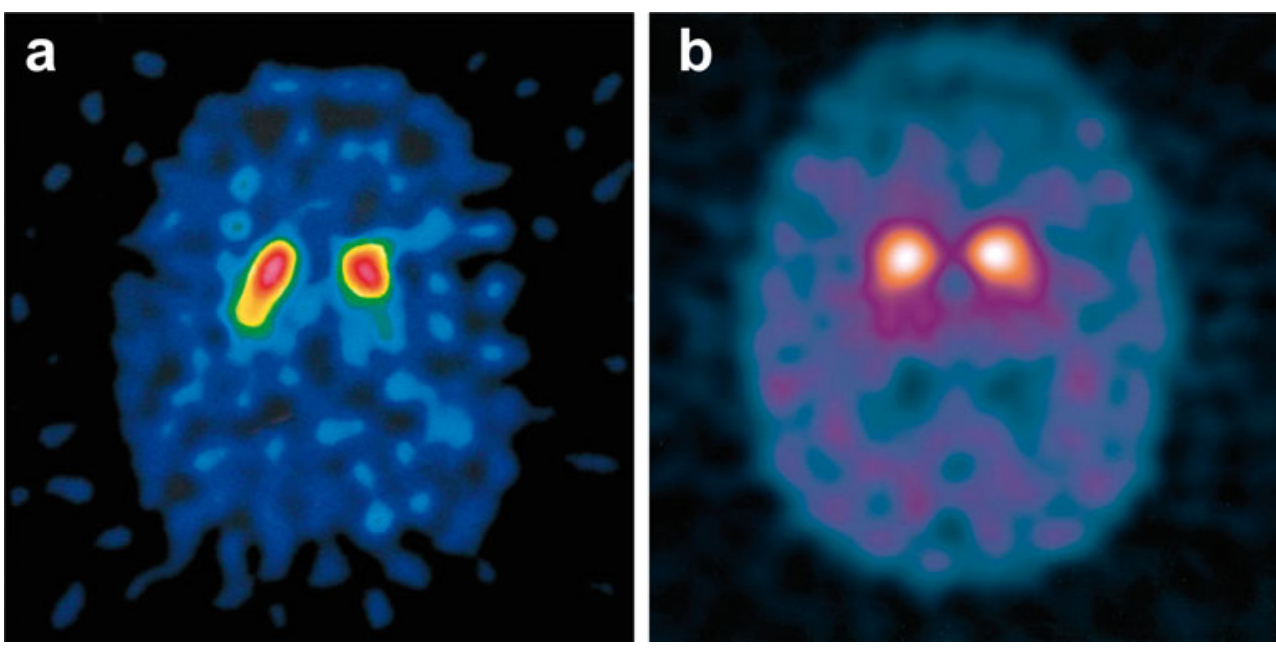

was 2 and the UPDRS motor score 28. Her MMSE was 30/ 30. At that time, she responded well to L-dopa treatment and no atypical features or additional neurological signs were observed; her clinical picture was therefore compatible with a diagnosis of PD. She died 3 years later, at the age of 78 , of acute myocardial infarction.

\section{Discussion}

Genetically isolated populations are an important resource for finding genetic determinants of human diseases. As a result of a limited number of founder alleles, prolonged geographical isolation, and genetic drift, the genetic architecture of disease is expected to be simpler in the genetic isolates than in the general populations, thereby facilitating the identification of the disease-causing or disease-predisposing alleles [25]. The population of Sardinia is one of the most extensively studied genetic isolates, which has yielded founder mutations and risk alleles in several disorders ([18, 19, 23, 26] and references therein).

The recent identification of the founder TARDBP $\mathrm{p}$. Ala382Thr mutation as the cause of a large percentage of ALS cases in this island is not only another example of the power of this approach. It is also a proof of principle that large etiological fractions of common neurodegenerative diseases can be underlain by rare mutations of large effects. A similar scenario has been delineated for the founder $p$. Gly2019Ser mutation in the LRRK2 gene as a cause of PD among patients of North African and Middle Eastern ancestries (reviewed in [27]).

The fact that all the three apparently healthy controls included in our study, who carried the p.Ala382Thr mutation, have developed ALS or dementia themselves or among their close relatives is a clear confirmation of the strong association between this mutation and the motoneuron disease-frontotemporal dementia spectrum. Furthermore, one control indi- vidual remains free from neurodegenerative signs and symptoms at the age of 79 years, illustrating the age-related, incomplete penetrance, already reported for this and other TARDBP mutations. A penetrance of $60 \%$ by age 70 has been recently estimated for the p.Ala382Thr mutation [19]. The incomplete penetrance is also in keeping with the frequent occurrence of this founder mutation among patients with sporadic disease presentation.

While TARDBP mutations have been detected in several patients with familial and sporadic ALS (approximately 2$3 \%$ of cases in large studies), to our knowledge, an association between TARDBP mutations and FTLD has been reported so far in only eight unrelated cases [4-6, 28, 29]. In two of these patients, the FTLD phenotype (familial in one and sporadic in the other case) was followed by the development of MND within 2 years [5]; in another three cases with familial ALS (and carrying the p.Ala382Thr mutation), the MND was the presenting phenotype, which was followed soon by an FTLD [29]. In the remaining three patients, sporadic FTLD presented as an isolated syndrome, without the development of MND within the follow-up period $[4,6,28]$. Of note, none of these cases presented parkinsonism.

Only three of the recently reported Sardinian ALS patients who carried the p.Ala382Thr mutation developed akinetic parkinsonism after the onset of ALS [18]. Therefore, our family with FTLD and parkinsonism (Fig. 1) represents already a further expansion of the clinical spectrum associated with TARDBP mutations, by showing two patients with FTLD and prominent parkinsonism without MND, and a third with pure, L-doparesponsive parkinsonism, in the absence of cognitive or motoneuron disturbances.

However, the most important finding of this study is the association between the p.Ala382Thr mutation and PD within our consecutive large Sardinian series. This points to a broader clinical expressivity of this mutation than earlier realized. 
To our knowledge, only one systematic study of the entire TARDBP coding region in $125 \mathrm{PD}$ patients of French-Canadian ancestry has been published, with negative results [30]. It is interesting to note that none of our eight PD patients who carry the mutation reported a family history for PD, ALS, or dementia, providing further evidence for the incomplete penetrance of this mutation.

Unfortunately, pathological studies are not available to verify whether Lewy bodies, TDP-43-positive inclusions, or both, are present in the brain of these patients. This remains a very important objective for follow-up studies. However, all these eight cases fulfill the widely accepted clinical diagnostic criteria for definite PD, including several supporting features (asymmetric onset, good and prolonged L-dopa response, presence of resting tremor, progressive course with the absence of atypical signs for more than 5 years). In two patients carrying the TARDBP p.Ala382Thr mutation, the DaTSCAN-SPECT imaging showed asymmetric defects, predominantly at the level of the putamen, as typically observed in idiopathic PD (Fig. 2). Apart from a slightly (and non-significantly) later onset age of $\mathrm{PD}$ symptoms, these cases were clinically indistinguishable from the other patients in our study.

The reasons of the broad variability in the clinical expression of this mutation remain to be found. It is possible that the severity and the rapid progression of the ALS hamper the detection of extrapyramidal signs. However, three patients who carried the p.Ala382Thr mutation described in one of the previous studies developed extrapyramidal symptoms several years after their initial presentation with motoneuron disease [18]. On the other hand, it is possible that the clinical signs of PD interfere with the detection of initial motoneuron disease signs. Nevertheless, none of our PD mutation carriers nor any of their close relatives developed clinical signs of the ALS spectrum.

It is likely that additional genetic (or non-genetic) factors modify the effects of the primary mutation (p.Ala382Thr), thereby leading to different clinical outcomes: ALS, FTLD, or parkinsonism, alone or in combination. For example, patients who manifest PD, or FTLD, as the resulting isolated clinical phenotype might carry mutations or polymorphisms in other loci that prevent (or delay) the onset of motoneuron disease and lead to the appearance of extrapyramidal or cognitive signs as the earliest clinical expression of the disease. Further work remains therefore ahead to identify the modifier(s) of this mutation, and genetic isolates (such as the Sardinian population) represent once more an ideal resource in this endeavor.

Following the initial association with the pathological hallmark inclusions in patients with FTLD, ALS, and ALSMND, the TDP-43 protein has also been detected in the pathological lesions in relevant percentages of brains of patients with Lewy body disorders, ranging from 7\% of PD cases to $19 \%$ of PD with dementia in one study [31], and to more than $50 \%$ of the brains with diffuse Lewy body disease in another study [32]. Moreover, pathological lesions containing the TDP-43 protein were reported in proximity of small blood vessels in the brain of one patient with familial Lewy body disease caused by the p.Ala53Thr mutation in the alpha-synuclein (SNCA) gene [33]. Taken together, these findings provide evidences of important intersections between the pathogenesis of the TDP-43 proteinopathies and the alpha-synucleinopathies, supporting the contention that TARDBP mutations might underlie prominent parkinsonism in patients with FTLD and also present as isolated PD.

In conclusion, our data provide a first direct link between the TARDBP mutation and PD, supporting an involvement of the TDP-43 protein in a broader neurodegenerative spectrum, including not only motoneuron disease and FTLD, but also PD. Further analyses of the whole TARDBP coding region in large series of familial and sporadic PD patients are warranted.

Acknowledgments We thank all the participating subjects for their contribution; Dr. Francesco Cucca (Institute of Neurogenetics and Neuropharmacology, Consiglio Nazionale delle Ricerche, Monserrato, Sardinia) for having generously contributed DNA samples from Sardinian control subjects; and Tom de Vries Lentsch, Erasmus $M C$, Rotterdam, for the artwork. This study was supported by grants from the "Internationaal Parkinson Fonds"-The Netherlands and the Netherlands Organization for Scientific Research (NWO, VIDI grant) to V.B.

Disclosure All experiments comply with the current laws of the countries in which they were performed. The authors declare that they have no conflict of interest.

Open Access This article is distributed under the terms of the Creative Commons Attribution Noncommercial License which permits any noncommercial use, distribution, and reproduction in any medium, provided the original author(s) and source are credited.

\section{References}

1. Neumann M, Sampathu DM, Kwong LK et al (2006) Ubiquitinated TDP-43 in frontotemporal lobar degeneration and amyotrophic lateral sclerosis. Science 314:130-133

2. Sreedharan J, Blair IP, Tripathi VB et al (2008) TDP-43 mutations in familial and sporadic amyotrophic lateral sclerosis. Science 319:1668-1672

3. Kabashi E, Valdmanis PN, Dion P et al (2008) TARDBP mutations in individuals with sporadic and familial amyotrophic lateral sclerosis. Nat Genet 40:572-574

4. Borroni B, Bonvicini C, Alberici A et al (2009) Mutation within TARDBP leads to frontotemporal dementia without motor neuron disease. Hum Mutat 30:E974-E983

5. Benajiba L, Le Ber I, Camuzat A et al (2009) TARDBP mutations in motoneuron disease with frontotemporal lobar degeneration. Ann Neurol 65:470-473 
6. Kovacs GG, Murrell JR, Horvath S et al (2009) TARDBP variation associated with frontotemporal dementia, supranuclear gaze palsy, and chorea. Mov Disord 24:1843-1847

7. Geser F, Martinez-Lage M, Robinson J et al (2009) Clinical and pathological continuum of multisystem TDP-43 proteinopathies. Arch Neurol 66:180-189

8. Ou SH, Wu F, Harrich D, Garcia-Martinez LF, Gaynor RB (1995) Cloning and characterization of a novel cellular protein, TDP-43, that binds to human immunodeficiency virus type 1 TAR DNA sequence motifs. J Virol 69:3584-3596

9. Buratti E, Brindisi A, Giombi M, Tisminetzky S, Ayala YM, Baralle FE (2005) TDP-43 binds heterogeneous nuclear ribonucleoprotein $\mathrm{A} / \mathrm{B}$ through its $\mathrm{C}$-terminal tail: an important region for the inhibition of cystic fibrosis transmembrane conductance regulator exon 9 splicing. J Biol Chem 280:37572-37584

10. Yokoseki A, Shiga A, Tan CF et al (2008) TDP-43 mutation in familial amyotrophic lateral sclerosis. Ann Neurol 63:538-542

11. Gitcho MA, Baloh RH, Chakraverty S et al (2008) TDP-43 A315T mutation in familial motor neuron disease. Ann Neurol 63:535-538

12. Van Deerlin VM, Leverenz JB, Bekris LM et al (2008) TARDBP mutations in amyotrophic lateral sclerosis with TDP-43 neuropathology: a genetic and histopathological analysis. Lancet Neurol 7:409-416

13. Rutherford NJ, Zhang YJ, Baker M et al (2008) Novel mutations in TARDBP (TDP-43) in patients with familial amyotrophic lateral sclerosis. PLoS Genet 4:e1000193

14. Kuhnlein P, Sperfeld AD, Vanmassenhove B et al (2008) Two German kindreds with familial amyotrophic lateral sclerosis due to TARDBP mutations. Arch Neurol 65:1185-1189

15. Corrado L, Ratti A, Gellera C et al (2009) High frequency of TARDBP gene mutations in Italian patients with amyotrophic lateral sclerosis. Hum Mutat 30:688-694

16. Del Bo R, Ghezzi S, Corti S et al (2009) TARDBP (TDP-43) sequence analysis in patients with familial and sporadic ALS: identification of two novel mutations. Eur J Neurol 16:727-732

17. Daoud H, Valdmanis PN, Kabashi E et al (2009) Contribution of TARDBP mutations to sporadic amyotrophic lateral sclerosis. J Med Genet 46:112-114

18. Chio A, Borghero G, Pugliatti M et al (2011) Large proportion of amyotrophic lateral sclerosis cases in Sardinia due to a single founder mutation of the TARDBP gene. Arch Neurol 68:594-598

19. Orru S, Manolakos E, Orru N et al (2011) High frequency of the TARDBP p.Ala382Thr mutation in Sardinian patients with amyotrophic lateral sclerosis. Clin Genet. doi:10.1111/j.13990004.2011.01668.x
20. Qureshi AI, Wilmot G, Dihenia B, Schneider JA, Krendel DA (1996) Motor neuron disease with parkinsonism. Arch Neurol 53:987-991

21. Hughes AJ, Daniel SE, Kilford L, Lees AJ (1992) Accuracy of clinical diagnosis of idiopathic Parkinson's disease: a clinicopathological study of 100 cases. J Neurol Neurosurg Psychiatry 55:181-184

22. Fahn S, Elton RL, Members of the UPDRS Development Committee (1987) Unified Parkinson's Disease Rating Scale. Recent developments in Parkinson's disease. Macmillan, New York, pp 153-163

23. Sanna S, Pitzalis M, Zoledziewska M et al (2010) Variants within the immunoregulatory CBLB gene are associated with multiple sclerosis. Nat Genet 42:495-497

24. Cossu G, van Doeselaar M, Deriu M et al (2007) LRRK2 mutations and Parkinson's disease in Sardinia - a Mediterranean genetic isolate. Parkinsonism Relat Disord 13:17-21

25. Heutink P, Oostra BA (2002) Gene finding in genetically isolated populations. Hum Mol Genet 11:2507-2515

26. Contu D, Morelli L, Santoni F, Foster JW, Francalacci P, Cucca F (2008) Y-chromosome based evidence for pre-neolithic origin of the genetically homogeneous but diverse Sardinian population: inference for association scans. PLoS One 3:e1430

27. Bonifati V (2007) LRRK2 low-penetrance mutations (Gly2019Ser) and risk alleles (Gly2385Arg)-linking familial and sporadic Parkinson's disease. Neurochem Res 32:1700-1708

28. Borroni B, Archetti S, Del Bo R et al (2010) TARDBP mutations in frontotemporal lobar degeneration: frequency, clinical features, and disease course. Rejuvenation Res 13:509-517

29. Chio A, Calvo A, Moglia C et al (2010) Amyotrophic lateral sclerosis-frontotemporal lobar dementia in 3 families with $p$. Ala382Thr TARDBP mutations. Arch Neurol 67:1002-1009

30. Kabashi E, Daoud H, Riviere JB et al (2009) No TARDBP mutations in a French Canadian population of patients with Parkinson disease. Arch Neurol 66:281-282

31. Nakashima-Yasuda H, Uryu K, Robinson J et al (2007) Comorbidity of TDP-43 proteinopathy in Lewy body related diseases. Acta Neuropathol 114:221-229

32. Arai T, Mackenzie IR, Hasegawa M et al (2009) Phosphorylated TDP-43 in Alzheimer's disease and dementia with Lewy bodies. Acta Neuropathol 117:125-136

33. Lin WL, Castanedes-Casey M, Dickson DW (2009) Transactivation response DNA-binding protein 43 microvasculopathy in frontotemporal degeneration and familial Lewy body disease. J Neuropathol Exp Neurol 68:1167-1176 